\title{
Statistical Forbidden Zone Data Hiding Technique to Secure Various Data File
}

\author{
Jayden Gabb, Sophia Brereton
}

\begin{abstract}
Video Steganography is a method where we can hide a wide range of documents with any extension into a carrying Video document. Right now exposition we are utilizing Forbidden Zone Data Hiding system where no adjustment is required in have signal range during information concealing procedure. Approach of this paper depends on the idea of message taking cover behind the video record that utilizations Forbidden Zone Data Hiding technique strategy. RA codes are utilizing with Forbidden zone information hiding to give robustness.
\end{abstract}

Keywords: Embedding, RA codes, Frames etc.

\section{INTRODUCTION}

This methodology gives an extreme secure procedure of information hiding utilizing spread document. This procedure utilizes various advances to getting an appropriate secure exchange between two parties. Henceforth, it very well may be expressed that unique commitment of this paper is to devise a complete video information hiding strategy.

\section{II. $\quad$ FZDH}

A) Architectural Framework

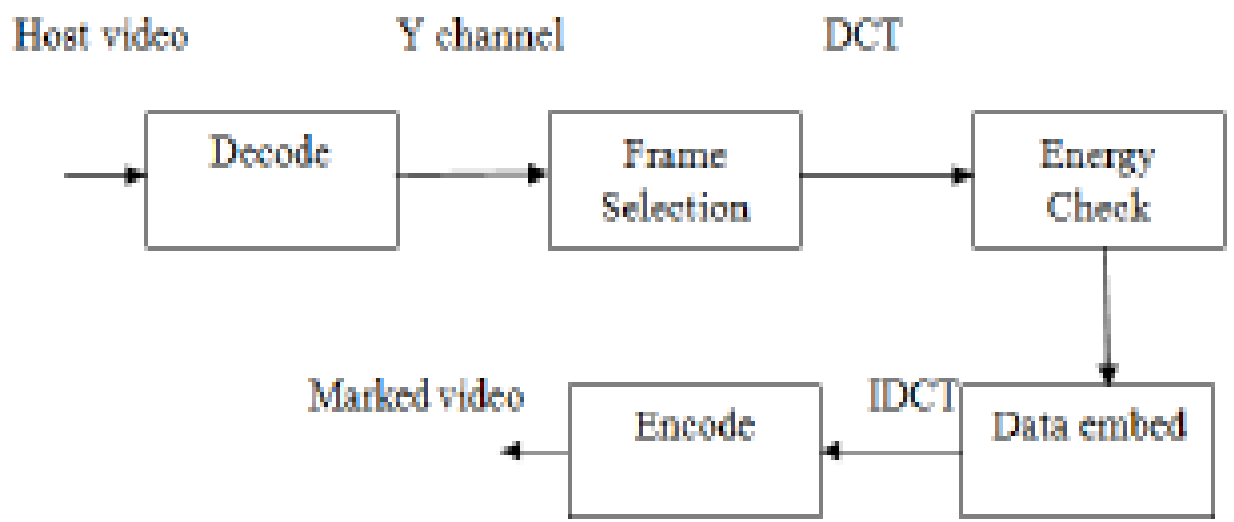

Fig. 1: Embedding Process 
The above figure delineates the implanting activity for asingle frame. Y-channel is being used for data embedding. This can be comprehended step by step as follows:

- In the initial step, frame selection is performed and the selected frames areprocessed blockwise.

- In each block, just a single bit is covered up. In the wake of acquiring $8 \times 8$ DCT of the square, energy check is performed on the coefficients that are predefined in a mask.

- Selected coefficients of variable length are utilized to hidedata bit $\mathrm{m}$. $\mathrm{m}$ is a message bits or frame synchronization markers.

- Message arrangement of each group is gotten by utilizing RA codes for $\mathrm{T}$ successive frames.

- Each and every square is alloted to one of these group toward the start.

- The inverse transform host frame is obtained.

The decoder flowchart is as per the following:

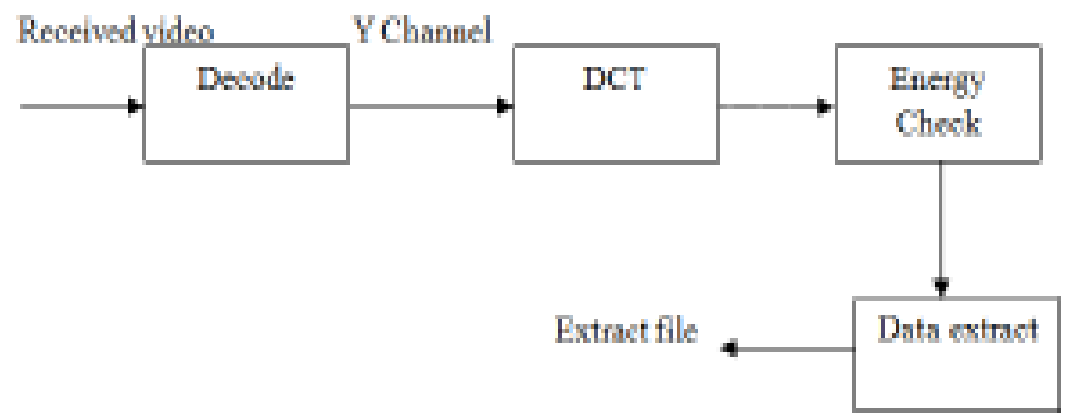

Fig. 2: Decoder

The contribution to decoder is the gotten video. Different stages associated with the procedure are:

1. DCT

2. Energy Check

3. Data Extraction

The procedure spoke to here is just for single edge. This is rehashed for other outlines in a similar way. Decoder can be twofold of implanted, with the special case that outline 
determination isn't performed. Edge synchronization markers are utilized for stamping outlines. Decoder utilizes the same framework parameters. The checked sign is dictated by decoder and afterward these values are sustained to the following stage which is information extraction. Deletions are the non-chose squares.

\section{B) Selective Embedding}

Specific inserting is a procedure which utilizes versatile host signal inspecting. The determination is acted in four phases, specifically:

1. Determination of edge

2. Assurance of recurrence band

3. Determination of square

4. Determination of coefficient.

These stages are talked about in detail beneath:

\section{A. Frame selection:}

In the entire frame chosen number of squares is checked. On the off chance that the proportion of chose squares to all squares is over a specific worth (T0), at that point outline is handled, something else, the frame is skipped.

\section{B. Frequency band:}

Figure 4.3 shows the center recurrence band of DCT coefficients. Just certain DCT coefficients are used.

\section{Block selection:}

For square determination vitality of the coefficients in the mask is determined. In the event that the vitality of the square is over a specific worth (T1) at that point the square is prepared, else, it is skipped.

\section{Coefficient choice:}

Right now is done between vitality of every coefficient and another limit T2. In the event that vitality is more than T2, all things considered it utilizes information installing together with other specific coefficients in the comparable square.

The stages are talked about underneath stepwise:

A. Right off the bat, the chose number of squares are checked dependent on the threshold value.

B. At that point, coefficiency is used.

C. The keep going stage centers around the determination of coefficient dependent on the energy of different squares and by comparing thresholds. It figures the energy of the coefficients in the mask. 


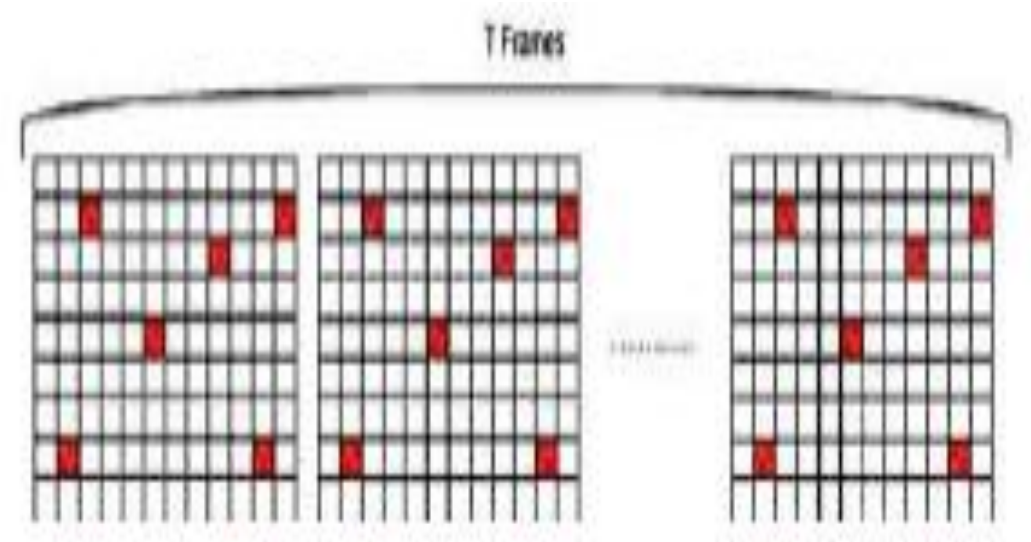

Fig.3 Typical Block Partitioning

C) Block Partitioning

Square apportioning capacities by making two disjoint implanted informational indexes which are message bits (m1) and edge synchronization markers (m2). An arbitrary key is created to decide the square areas of $\mathrm{m} 2$. Message bits (m1) use the remainder of the frame. A similar dividing is accomplished for all frame. Edge synchronization markers $(\mathrm{m} 2)$ are inserted outline by outline. Then again, $\mathrm{m} 1$ is scattered to $\mathrm{T}$ sequential frames. Them two are gotten as the results of the RA encoder.

D) Erasure Handling

Right now, codes are utilized to decide the chose obstructs at the embedder. Square choice is versatile in nature, the theme of utilizing RA codes is on the grounds that it is important to decide obstructs in embedder while de-synchronization is being performed between the embedder and decoder.

To evade non-assurance of chose hinders at the embedder by decoder during implanting activity, blunder adjustment codes strong to eradications, for example, RA codes are utilized in picture and video information covering up in past endeavors. 


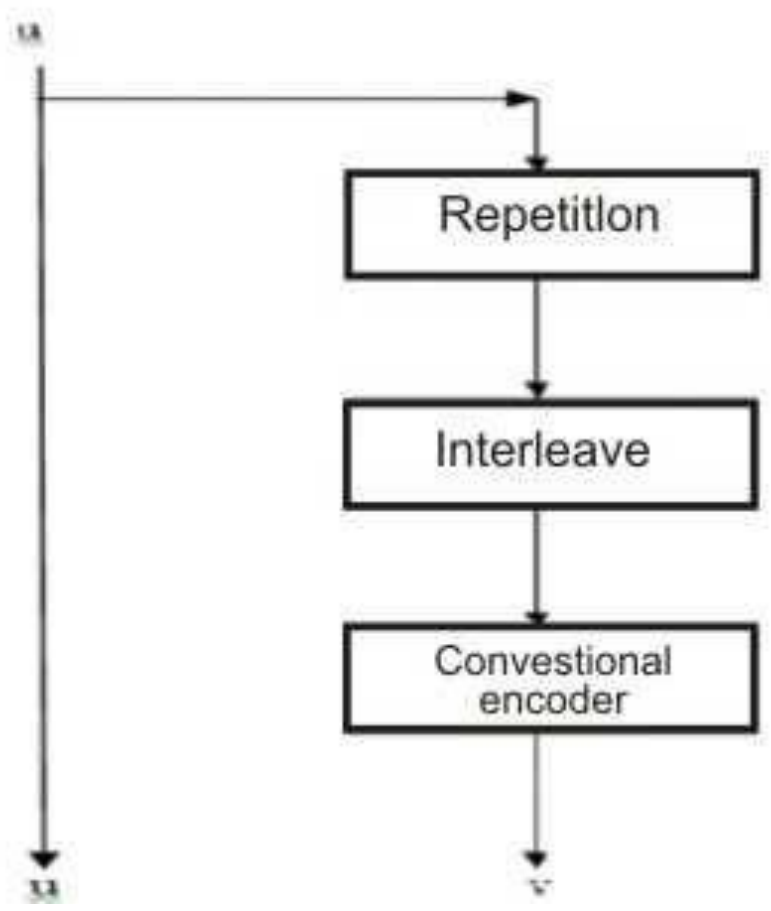

Fig. 4 RA encoder

As appeared in Figure info is set toward the start of the yield. Right now paper, we are concentrating on usage of precise RA codes to acquire $m 1$ as $u 1+v 1$ and $m 2$ as $u 2+v 2$. Here,

- $\mathrm{u} 1$ indicates the encoded message bits and

- $\mathrm{u} 2$ is the encoded outline synchronization marker bits.

E) Frame Synchronization

Right now, for example, neighborhood outline file beginning from 0 to $\mathrm{T}-1$ are appointed to each frame inside a gathering of $\mathrm{T}$ back to back edges. The advantage of utilizing thesemarkers is:

- These can be utilized to decide the frame drops, embeds and rehashes, just as the finish of the gathering of frames so, all things considered all fundamental message bits are accessible for RA decoder.

- K2 bits speak to outline lists. RK2 bits are gotten after RA encoders which are saved for outline markers. $\mathrm{K} 2>\log 2 \mathrm{~T}$, so a little $2 \mathrm{k} 2$ part of code words is legitimate. Consequently, a legitimate frame can be distinguished with higher likelihood.

Advantages of successive edge file data are as per the following:

- Increase power. 
- RA code spreads the yield code expressions of the adjoining outline files which prompts less mistakes event when unraveling contiguous edge lists. Once one stores RK2 hinders for outline markers, $\mathrm{T}(\mathrm{N}-\mathrm{RK} 2)$ squares stay for message bits.

\section{F) Decoding}

This procedure can be clarified stepwise as follows:

1) An information structure of length RK1 is saved for channel perception likelihood values, om at the decoder end.

2) Then introduction of structure with deletions ( $\mathrm{m}=0.5$ for $\mathrm{m}=0$ and $\mathrm{m}=1$ ) is done.

3) During the decipher procedure outline synchronization markers are decoded first at each frame.

4) Once the finish of the gathering of frames is recognized, message interpreting is performed.

5) Two edge file esteems are put away: current and past files where current outline and past frame signify the present and past frame records, separately.

At that point the accompanying guidelines are utilized to interpret $\mathrm{u} 1$.

- If current edge $>\mathrm{T}$, at that point avoid this edge. (This case relates to unmarked frame)

- If current edge = previous frame, at that point skirt this edge. (This case relates to frame repeat)

- Else, process the present edge. Put om values in the comparing spot of the information structure. Right now squares are left as erasures.

- If current edge < past edge, this implies the finish of gathering of outlines is come to, consequently now decipher the message bits and get $\mathrm{u} 1$ and afterward instate data structure.

\section{CONCLUSION}

To safely transferring the data record, we use video information covering up and utilizing amendment limit of rehash aggregate code with prevalence of FZDH. Essentially we concentrating on enormous video record that size bigger than a hide record is additionally safely covered up with no information misfortune. The primary bit of leeway of utilizing video record sequestered from everything data is the additional protection from of the outsider or unintended beneficiary because of the general multifaceted nature of video contrasted with picture and sound record. This framework is useful for the protection and security divisions sending and getting the secret issues in emergency circumstances. 


\section{REFERENCES}

[1] K. Sullivan, Z. Bi, U. Madhow, S. Chandrasekaran, B.S. Manjunath,” Steganalysis of Quantization Index Modulation Data Hiding", Dept. of Electrical andComputer Engineering University of California at Santa Barbara CA 93106

[2] ResojuOmprakash , D. Jyothi," Block Based Adaptive Videodata Hiding Technique ", International Journal of Mathematical Sciences, Technology and Humanities 70 (2012) 789 - 797, ISSN 2249-5460

[3] A.Hamsathvani," Image Hiding in Video Sequence Based On MSE",International Journal of Electronics and Computer Science Engineering, ISSN- 2277-1956

[4] Dr. P. R. Deshmukh ,BhagyashriRahangdale,” Data Hiding using Video Steganography” ,nternational Journal of Engineering Research \& Technology (IJERT) Vol. 3 Issue 4, April - 2014.

[5] RichaKhare, Dr. KuldeepRaghuwanshi "” A Review Of Video Steganography Methods", International Journal of Research in Advent Technology Volume 2, Issue 1, January 2014

[6] KalaiselvanSA ,Karthick V," Robust Video Data Hiding at Forbidden Zone Using Stegnography", National Conference on Advances in Computer Science and Information Technology, ISBN:978-81924031-8-2

[7] Poonam V Bodhak, Baisa L Gunjal," Improved Protection In Video Steganography Using DCT \& LSB”, International Journal of Engineering and Innovative Technology (IJEIT) Volume 1, Issue 4, April 2012

[8] S.Chitra, NarasimhaluThoti," Implementation of Video Steganography UsingHash Function in LSB Technique", International Journal of Engineering Research \&Technology (IJERT) Vol. 2 Issue 11, November -2013

[9] Min Wu, Member, Heather Yu, Bede Liu," Data Hiding in Image and Video: Part II—Designs and Applications", IEEE Transactions On Image Processing, Vol. 12, No. 6, June 2003

[10] J. J. Chae , B. S. Manjunath," Data Hiding in Video", Department of Electrical and Computer Engineering University of California, Santa Barbara CA 93106-9560 .

[11] Min Wu , Bede Liu ," Data Hiding in Image and Video: Part I-Fundamental Issues and Solutions" IEEE Transactions On Image Processing, Vol. 12 (6), 2003.

[12] Arvind Kumar, Km. Pooja, "Steganography- A Data Hiding Technique" International Journal of Computer Application, Vol. 9, No.7, November 2010. 